\title{
ON THE STATOR FLUX LINKAGE ESTIMATION OF AN PMSM WITH EXTENDED KALMAN FILTERS
}

\author{
T.J. Vyncke, R.K. Boel, and J.A.A. Melkebeek \\ Department of Electrical Energy, Systems and Automation (EESA) \\ Ghent University (UGent), Sint-Pietersnieuwstraat 41, B-9000 Gent, Belgium \\ phone: +32 (0)9 264 3442, fax: +32 (0)9 2643582 e-mail: Thomas.Vyncke@UGent.be
}

Keywords: PMSM, extended kalman filter, stator flux estimation, DTC, parameter estimation, saturation

\begin{abstract}
The demand for drives with high quality torque control has grown tremendously in a wide variety of applications. Direct torque control (DTC) for permanent magnet synchronous motors can provide this accurate and fast torque control. When applying DTC the change of the stator flux linkage vector is controlled. As such the estimation of the stator flux linkage is essential. In this paper the performance of the Extended Kalman Filter (EKF) for stator flux linkage estimation is studied. Starting from a formulation of the EKF for isotropic motors, the influence of rotor anisotropy and saturation is evaluated. Subsequently it is expanded to highly isotropic motors as well. In both cases the possibilities to add parameter estimations are evaluated.
\end{abstract}

\section{Introduction}

In recent years the use of highly dynamic electrical drives has increased steadily in applications ranging from industry automation to electrical cars. These highly dynamic electrical drives have to provide accurate and fast torque control together with the highest possible efficiency. Owing to their characteristics of high efficiency, high power density and reliability AC machines, and more recently especially permanent magnet synchronous machines (PMSM's), have obtained dominance. To control the torque and flux levels of $\mathrm{AC}$ machines rotor flux oriented control is seen as an industry standard. Alternatives however exist and Direct Torque Control (DTC) can provide accurate fast torque control. Originally proposed for induction motors in [7] DTC became rather popular in the past two decades. This is mainly due to the fact that DTC for induction machines is inherently motion-state sensorless. This lead several authors [5], [6], [9] to propose ways to adapt DTC to work with PMSMs.

The principles of DTC are derived by examining the equation for electromagnetic torque $T$ of an PMSM

$$
T=\frac{3}{4} \frac{N_{p}\left|\underline{\Psi}_{s}\right|}{L_{d} L_{q}}\left(2\left|\underline{\Psi}_{f}\right| L_{q} \sin \delta-\left|\underline{\Psi}_{s}\right|\left(L_{q}-L_{d}\right) \sin 2 \delta\right),
$$

where $\delta$ denotes the load angle between the stator flux linkage $\underline{\Psi}_{s}$ and permanent magnet flux linkage $\underline{\Psi}_{f}$ vectors in the stationary $\alpha \beta$ frame. The number of pole pairs is denoted by $N_{p} . L_{d}$ and $L_{q}$ are the direct and quadrature axis inductances. If these inductances are equal, the equation (1) reduces to (2). This is often assumed for an PMSM with surface-mounted magnets where the direct and quadrature axis reluctances are almost identical and thus $L_{d}=L_{q}=L_{s}$ is used.

$$
T=\frac{3 N_{p}}{2 L_{s}}\left|\underline{\Psi}_{f}\right|\left|\underline{\Psi}_{s}\right| \sin \delta
$$

From (1-2) can be seen that for constant stator flux linkage, the torque is changed by changing the load angle $\delta$. The stator flux vector can be changed by applying from the inverter the voltage vector with the most appropriate radial and tangential components. Although different control strategies are used in DTC, in all implementations the switching decision is based on three estimated variables. These are: estimated torque $T=$ $\frac{3}{2} N_{p}\left(\Psi_{s, \alpha} I_{\beta}-\Psi_{s, \beta} I_{\alpha}\right)$, stator flux linkage magnitude $\left|\underline{\Psi}_{s}\right|$ and angle $\theta_{\Psi_{s}}$. As these are determined by the estimation of the stator flux linkage components $\Psi_{s, \alpha}$ and $\Psi_{s, \beta}$ the estimation is crucial for correct operation of the drive, as shown in figure 1.

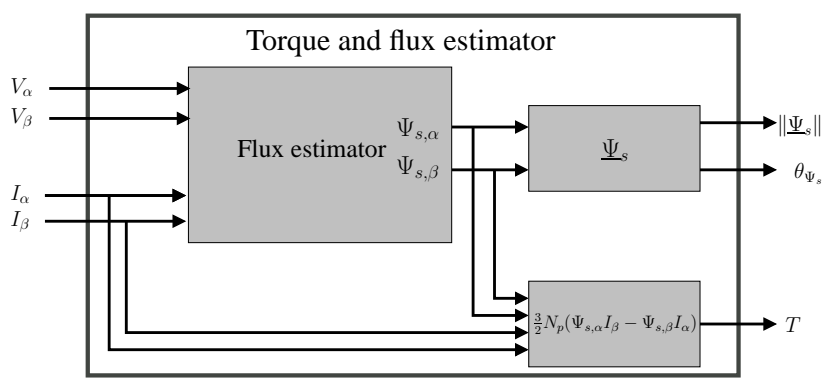

Figure 1: Torque and flux estimator schematic for DTC

Several estimation techniques have been reported in the literature [3]-[5], [9], a comparison is given in [10]. One possibility is the use of state observers, such as the Extended Kalman Filter $(E K F)$, that calculate the stator flux linkage vector with estimated state variables (including rotor speed and position). The EKF is often discussed for the sensorless control of PMSMs, however focused on the sensorless position estimation for current control in the rotor flux reference frame. Few publications discuss the EKF for the estimation of the stator flux linkage vector [3], [4], [10], [11].

In this paper EKFs for stator flux linkage estimation are discussed in section 2, with different state vectors for an SPMSM. Estimation errors due to incorrect motor parameters are addressed and the EKFs are expanded to include parameter estimations. In section 3 the effects of neglecting isotropy in the rotor are studied, saliency and saturation in both the $d$ and $q$ axis of an SPMSM are considered. To further improve the estimations and to expand the field of applications to motors with large saliency, in section 4 different formulations of the EKF for motors with anisotropic rotors are given and compared. 


\section{Reduced-order EKF for isotropic PMSM}

\subsection{Stator Flux Linkage Estimation}

In theory the integration of the back-emf can be used for this estimation when stator voltages and currents are measured:

$$
\underline{\Psi}_{s}=\int_{0}^{t}\left(\underline{V}_{s}-R_{s} \underline{I}_{s}\right) d t+\underline{\Psi}_{s \mid t=0}
$$

The use of a pure open-loop integration is a simple method, relying on only one parameter $R_{s}$ and independent of the rotor position, but has many disadvantages. An overview and comparison of several improved methods based on this principle is given in [10], where also current model based methods are discussed and compared. The current model is defined in the rotor oriented $d q$ reference frame for anisotropic rotors by

$$
\begin{aligned}
& \Psi_{d}=L_{d} I_{d}+\Psi_{f} \\
& \Psi_{q}=L_{q} I_{q}
\end{aligned}
$$

or in the stationary $\alpha \beta$ reference frame for an SPMSM with isotropic rotor:

$$
\begin{aligned}
& \Psi_{s, \alpha}=L_{s} I_{\alpha}+\Psi_{f} \cos (\theta) \\
& \Psi_{s, \beta}=L_{s} I_{\beta}+\Psi_{f} \sin (\theta) .
\end{aligned}
$$

As is clear from equations (4-7), these methods are dependent on the rotor position $\theta$, stator inductance $\left(L_{d}\right.$ and $L_{q}$ or $\left.L_{s}\right)$ and permanent magnet flux $\Psi_{f}$. The resulting need for a position sensor is, especially in DTC which is an inherently position sensorless method, considered as a major disadvantage. Also the increased parameter dependence on the inductances is, considering the saturation, a disadvantage. To reduce the parameter dependence and to perform the rotor position estimation needed in the current model a state observer can be used.

\subsection{Reduced-order EKF}

The Kalman filter is a stochastic recursive optimum-state estimator. For nonlinear systems an extended Kalman filter (EKF) can be used to obtain unmeasurable states (e.g. speed and rotor position) by using a model for the dynamical system, measured states and statistics of the system and measurement noise. By means of the noise input it is possible to take account of both measuring errors and modelling errors. The EKF is a two-step method as shown in figure 2 . With the measured inputs $\mathbf{u}_{k}$ and machine model $(\mathbf{f}(\mathbf{x}, \mathbf{u})$ and $\mathbf{h}(\mathbf{x}))$ the next state of the machine $\hat{\mathbf{x}}_{k+1}$ is predicted (prediction step). From this state the next output $\hat{\mathbf{y}}_{k+1}$ is calculated and compared to the measured value $\mathbf{z}_{k+1}$. The error on the output, together with the covariance values of measurement noise $\mathbf{R}$ and system $\mathbf{Q}$ are used to correct the state values in the next step. Often the covariance matrices are chosen to be diagonal. In this correction or innovation step the Kalman gain matrix $\mathbf{K}_{k+1}$ is calculated as well.

In this paper two implementations of the EKF are studied. The same nonlinear state-space model for the PMSM is used, the difference between the two methods is based on the selection of the state variables.

\section{EKFC: EKF with current components}

In EKFC the current components in the stationary reference frame are selected as state variables, as in [4], [9]:

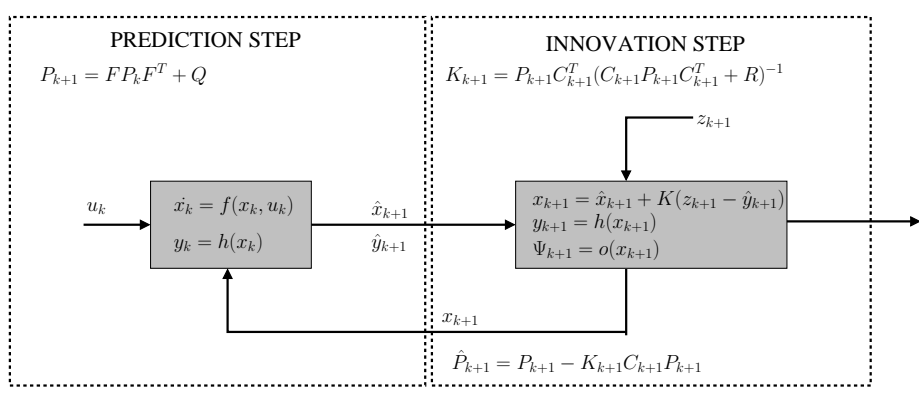

Figure 2: EKF scheme

$$
\mathbf{x}=\left[\begin{array}{llll}
x_{1} & x_{2} & x_{3} & x_{4}
\end{array}\right]^{T}=\left[\begin{array}{lll}
I_{\alpha} & I_{\beta} & \omega
\end{array}\right]^{T},
$$

Here $\omega$ and $\theta$ denote rotor speed and position respectively.

\section{EKFF: EKF with flux components}

The stator flux linkage components in the stationary reference frame are selected as state variables, as in [3]

$$
\mathbf{x}=\left[\begin{array}{llll}
x_{1} & x_{2} & x_{3} & x_{4}
\end{array}\right]^{T}=\left[\begin{array}{lll}
\Psi_{s, \alpha} & \Psi_{s, \beta} & \omega
\end{array}\right]^{T}
$$

In both cases the voltage $\mathbf{u}=\left[V_{\alpha} V_{\beta}\right]^{T}$ and current components in the stationary reference frame $\mathbf{y}=\left[\begin{array}{ll}I_{\alpha} & I_{\beta}\end{array}\right]^{T}$ are selected as input and output respectively. The EKF is of reduced order as the inertia is assumed to be infinite so that the mechanical equation is omitted. This is very advantageous as the load torque and inertia in the mechanical equation typically are not known. Because the speed $\omega$ is in the state vector the EKF will correct this modelling error if a good value is chosen for the covariance. More details about the tuning of EKF's can be found in [2].

For EKFC and EKFF respectively the system function $\mathbf{f}(\mathbf{x}, \mathbf{u})$, output function $\mathbf{h}(\mathbf{x})$ and Jacobians $\mathbf{F}=\frac{\mathbf{f}(\mathbf{x}, \mathbf{u})}{\partial \mathbf{x}}$ and $\mathbf{C}=\frac{\mathbf{h}(\mathbf{x})}{\partial \mathbf{x}}$ are given in Table I. When inspecting the Jacobians it is obvious that the expression of $\mathbf{F}$ is more complicated for EKFC than EKFF while the reverse is true for $\mathbf{C}$. In this paper we define, besides the output function $\mathbf{y}$ that is used to correct the estimation, an additional output function $\mathbf{o}(\mathbf{x})$ expressing the 'useful' output (stator flux components) as a function of the state components.

\subsection{Influence of parameter variations}

In [10] and [11] the effects of incorrect values for the stator resistance $R_{s}$, stator inductance $L_{s}$, and permanent magnet flux $\Psi_{f}$ on the performance of EKFC and EKFF in a DTC drive have been investigated. The influence of an incorrect value of the stator resistance $R_{s}$ is found to be very small and only important during transients, even for large deviations of $R_{s}$. This is true for both the error in estimated stator flux vector magnitude and angle. As the EKFs are based on the current model the dependence on $L_{s}$ and $\Psi_{f}$ is investigated as well. As shown in [10], the estimators remain stable but show both a dynamic and steady-state deviation of both flux magnitude and angle. The estimations with EKFC and EKFF in this case do not yield better results than the open-loop current model with measured position. The remaining advantage is a sensorless estimation.

The correction in the estimation when $R_{s}$ is varied is the result of the fact that the EKF estimators can correct for the modeling inaccuracies by the feedback loop. For variations in $L_{s}$ and $\Psi_{f}$ 


\begin{tabular}{|c|c|c|c|c|c|c|}
\hline EKFC & 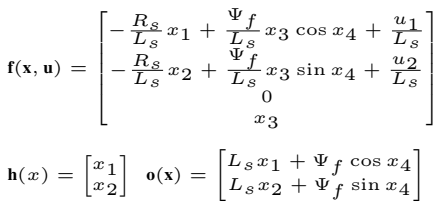 & 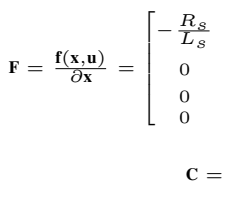 & $\begin{array}{r}0 \\
-\frac{R}{L} \\
0 \\
0 \\
\frac{\mathbf{h}(\mathbf{x})}{\partial \mathbf{x}}=\end{array}$ & 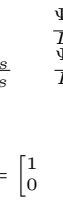 & 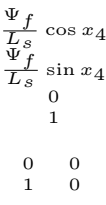 & $\left.\begin{array}{cc}4 & -\frac{\Psi f}{L_{s}} x_{3} \sin x_{4} \\
4 & \frac{\Psi_{f}}{L_{s}} x_{3} \cos x_{4} \\
0 \\
0\end{array}\right]$ \\
\hline \multirow{2}{*}{ EKFF } & $\mathbf{f}(\mathbf{x}, \mathbf{u})=\left[\begin{array}{c}-\frac{R_{s}}{L_{s}} x_{1}+\Psi_{f} \frac{R_{s}}{L_{s}} \cos x_{4}+u_{1} \\
-\frac{R_{s}}{L_{s}} x_{2}+\Psi_{f} \frac{R_{s}}{L_{s}} \sin x_{4}+u_{2} \\
0 \\
x_{3}\end{array}\right]$ & \multirow[t]{2}{*}{$\mathbf{F}=\frac{\mathbf{f}(\mathbf{x}, \mathbf{u})}{\partial \mathbf{x}}=$} & $\frac{R_{s}}{L_{s}}$ & $\begin{aligned} & 0 \\
&-\frac{R_{s}}{L_{s}} \\
& 0 \\
& 0\end{aligned}$ & $\begin{array}{l}0 \\
0 \\
0 \\
1\end{array}$ & $\left.\begin{array}{c}-\Psi_{f} \frac{R_{s}}{L_{s}} \sin x_{4} \\
\Psi_{f} \frac{R_{s}}{L_{s}} \cos x_{4} \\
0 \\
0\end{array}\right]$ \\
\hline & $\mathbf{h}(\mathbf{x})=\left[\begin{array}{l}\frac{x_{1}-\Psi_{f} \cos x_{4}}{L_{s} \sin x_{4}} \\
\frac{x_{2}-\Psi_{f} \sin x_{s}}{L_{s}}\end{array}\right] \quad \mathbf{o}(\mathbf{x})=\left[\begin{array}{l}x_{1} \\
x_{2}\end{array}\right]$ & & {$\left[\begin{array}{c}\frac{1}{L_{s}} \\
0\end{array}\right.$} & $\begin{array}{l}0 \\
\frac{1}{L_{s}}\end{array}$ & $\begin{array}{l}0 \\
0\end{array}$ & $\left.\begin{array}{l}\frac{\Psi_{f}}{L_{s}} \sin x_{4} \\
\frac{\Psi_{f}}{L_{s}} \sin x_{4}\end{array}\right]$ \\
\hline
\end{tabular}

Table I: Equations for EKFC and EKFF

\begin{tabular}{|c|c|c|c|c|c|c|}
\hline Name of EKF & Added state & & $x$ & $f(\mathbf{x}, \mathbf{u})$ & $\mathbf{h}(\mathbf{x})$ & $\mathbf{o ( x )}$ \\
\hline EKFCA1 & $L_{s}$ & {$\left[\begin{array}{l}x_{1} \\
x_{2} \\
x_{3} \\
x_{4} \\
x_{5}\end{array}\right]$} & $=\left[\begin{array}{c}I_{\alpha} \\
I_{\beta} \\
\omega \\
\theta \\
1 \\
\frac{1}{L_{s}}\end{array}\right]$ & 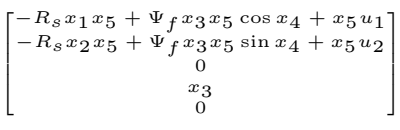 & {$\left[\begin{array}{l}x_{1} \\
x_{2}\end{array}\right]$} & {$\left[\begin{array}{l}\frac{x_{1}}{x_{5}}+\Psi_{f} \cos x_{4} \\
\frac{x_{2}}{x_{5}}+\Psi_{f} \sin x_{4}\end{array}\right]$} \\
\hline EKFCA3 & $L_{s}, R_{s}, \Psi_{f}$ & {$\left[\begin{array}{l}x_{1} \\
x_{2} \\
x_{3} \\
x_{4} \\
x_{5} \\
x_{6} \\
x_{7}\end{array}\right]$} & $=\left[\begin{array}{c}I_{\alpha} \\
I_{\beta} \\
\omega \\
\theta \\
\frac{1}{L_{s}} \\
R_{s} \\
\Psi_{f}\end{array}\right]$ & {$\left[\begin{array}{c}-x_{1} x_{5} x_{6}+x_{3} x_{5} x_{7} \cos x_{4}+x_{5} u_{1} \\
-x_{2} x_{5} x_{6}+x_{3} x_{5} x_{7} \sin x_{4}+x_{5} u_{2} \\
0 \\
x_{3} \\
0 \\
0 \\
0\end{array}\right]$} & {$\left[\begin{array}{l}x_{1} \\
x_{2}\end{array}\right]$} & {$\left[\begin{array}{l}\frac{x_{1}}{x_{5}}+x_{7} \cos x_{4} \\
\frac{x_{2}}{x_{5}}+x_{7} \sin x_{4}\end{array}\right]$} \\
\hline EKFFA1 & $L_{s}$ & {$\left[\begin{array}{l}x_{1} \\
x_{2} \\
x_{3} \\
x_{4} \\
x_{5}\end{array}\right]$} & $=\left[\begin{array}{c}\Psi_{s, \alpha} \\
\Psi_{s, \beta} \\
\omega \\
\theta \\
\frac{1}{L_{s}}\end{array}\right]$ & {$\left[\begin{array}{c}-R_{s} x_{1} x_{5}+R_{s} \Psi_{f} x_{5} \cos x_{4}+u_{1} \\
-R_{s} x_{2} x_{5}+R_{s} \Psi_{f} x_{5} \sin x_{4}+u_{2} \\
0 \\
x_{3} \\
0\end{array}\right]$} & {$\left[\begin{array}{l}x_{5}\left(x_{1}-\Psi_{f} \cos x_{4}\right) \\
x_{5}\left(x_{2}-\Psi_{f} \sin x_{4}\right)\end{array}\right]$} & {$\left[\begin{array}{l}x_{1} \\
x_{2}\end{array}\right]$} \\
\hline EKFFA3 & $L_{s}, R_{s}, \Psi_{f}$ & {$\left[\begin{array}{l}x_{1} \\
x_{2} \\
x_{3} \\
x_{4} \\
x_{5} \\
x_{6} \\
x_{7}\end{array}\right]$} & $=\left[\begin{array}{c}\Psi_{s, \alpha} \\
\Psi_{s, \beta} \\
\omega \\
\theta \\
1 \\
L_{s} \\
R_{s} \\
\Psi_{f}\end{array}\right]$ & {$\left[\begin{array}{c}-x_{1} x_{5} x_{6}+x_{5} x_{6} x_{7} \cos x_{4}+u_{1} \\
-x_{2} x_{5} x_{6}+x_{5} x_{6} x_{7} \sin x_{4}+u_{2} \\
0 \\
x_{3} \\
0 \\
0 \\
0\end{array}\right]$} & {$\left[\begin{array}{l}x_{5}\left(x_{1}-x_{7} \cos x_{4}\right) \\
x_{5}\left(x_{2}-x_{7} \sin x_{4}\right)\end{array}\right]$} & {$\left[\begin{array}{l}x_{1} \\
x_{2}\end{array}\right]$} \\
\hline
\end{tabular}

Table II: Equations for EKFC and EKFF with augmented state vector

this is not the case as these parameters, unlike $R_{s}$, are not only used in $\mathbf{f}(\mathbf{x}, \mathbf{u})$. For EKFC the state vector $\mathbf{x}$ will converge to the correct values, but due to the use of $L_{s}$ and $\Psi_{f}$ in $\mathbf{o}(\mathbf{x})$ to determine $\Psi_{\alpha}$ and $\Psi_{\beta}$ from $\mathbf{x}$ the output is incorrect (i.e. the same situation as for the current model with measured rotor position). For EKFF however $L_{s}$ and $\Psi_{f}$ are used in $\mathbf{h}(\mathbf{x})$ and thus the state vector $\mathbf{x}$ will not converge to the correct values.

\subsection{Adding parameter estimations to the EKF}

As discussed in [10], [11] the EKFs fail to estimate the stator flux vector correctly if motor parameters used in $\mathbf{h}(\mathbf{x})$ and $\mathbf{o}(\mathbf{x})$ deviate from the true values. A possible solution to overcome this problem is investigated in [11]. The most important motor parameters in the EKF are estimated as well. This can be done by augmenting the state vector with the parameters to be estimated, where parameter variations are given no dynamics (i.e. the corresponding row of $\mathbf{f}(\mathbf{x}, \mathbf{u})$ is 0$)$. In [11] three cases and aspects of the practical FPGA implementation are discussed.

Here two cases are considered, Table II gives the expressions for EKFC and EKFF with added parameter estimations. In the first case (EKFCA1 and EKFFA1) $L_{s}$ is added to estimate as this is a parameter that can vary strongly during operation of the drive. It has to be noted that in order to take the variation of $L_{s}$ into account, actually $\frac{1}{L_{s}}$ is added to the state vector. The choice for $L_{s}$ as a state component would, due to the partial differentiation, result in expressions for $\mathbf{F}$ and $\mathbf{C}$ that are much more complex (too complex for actual implementation).
In the second case (EKFCA3 and EKFFA3) the estimation of all three relevant parameters $L_{s}, R_{s}, \Psi_{f}$ is performed. For the sake of brevity, the expressions for the Jacobians $\mathbf{F}$ and $\mathbf{C}$ have been omitted in Table II. However it is clear that the increased complexity is even more easily seen in $\mathbf{F}$ and $\mathbf{C}$ due to the increased non-linearity of the model. This of course means that the computation of the estimation covariance matrix $\mathbf{P}$ and the Kalman correction matrix $\mathbf{K}$ become increasingly complex [11].

As shown in [11] adding parameters to the estimation can work, but great care should be taken as unwanted cross coupling effects of parameter variations can occur with a poorly chosen covariance matrix Q. Clearly it is important to refrain from putting too little confidence (high values in $\mathbf{Q}$ ) in the model as this could induce overcompensation of the parameters and thus result in poor performance. However with a good choice of covariance matrix good estimations can be obtained.

\section{Motor anisotropy and saturation}

Up till this point only isotropic motors have been considered, i.e. motors for which the reluctances along the direct and quadrature axes are equal. It is customary to consider permanent magnet synchronous motors with surface-mounted magnets (SPMSM) as completely isotropic motors since often a symmetrical nonsalient rotor construction is assumed and the magnet permeance is equal to that of air. Due to the relatively large air-gap saturation is often assumed to be negligible for SPMSMs. 


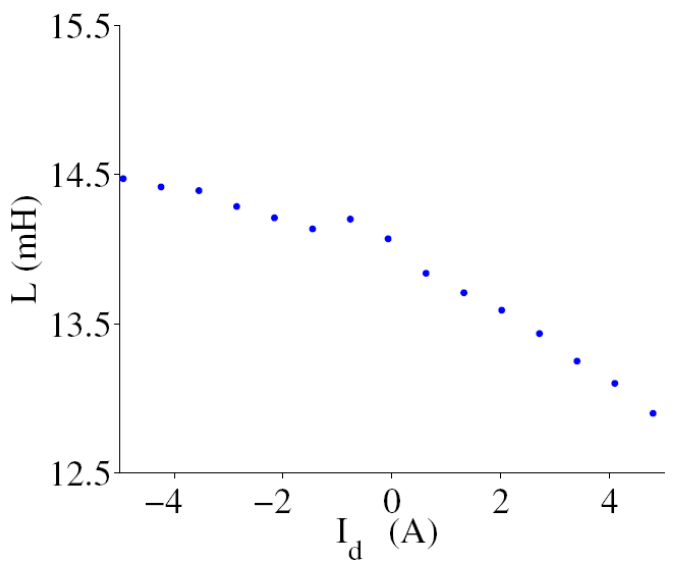

(a)

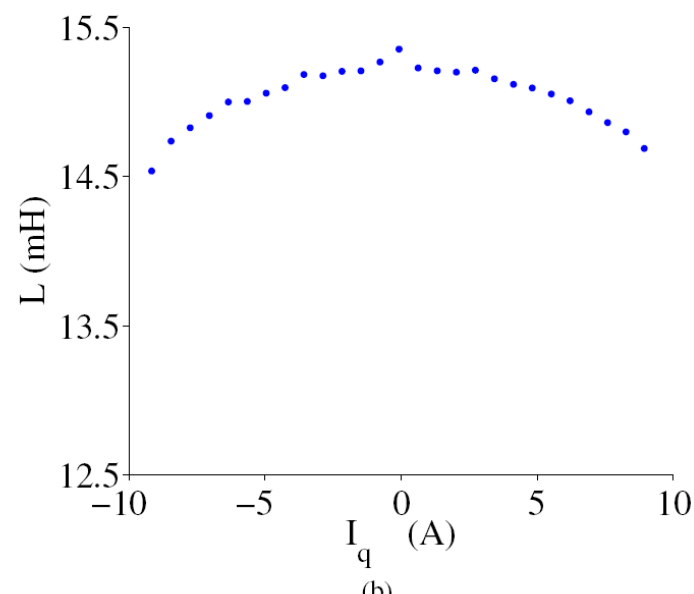

(b)

Figure 3: Obtained inductances for a Siemens PMSM, total inductances are shown: to obtain $L_{d}$ and $L_{q}$ divide by $\frac{3}{2}$
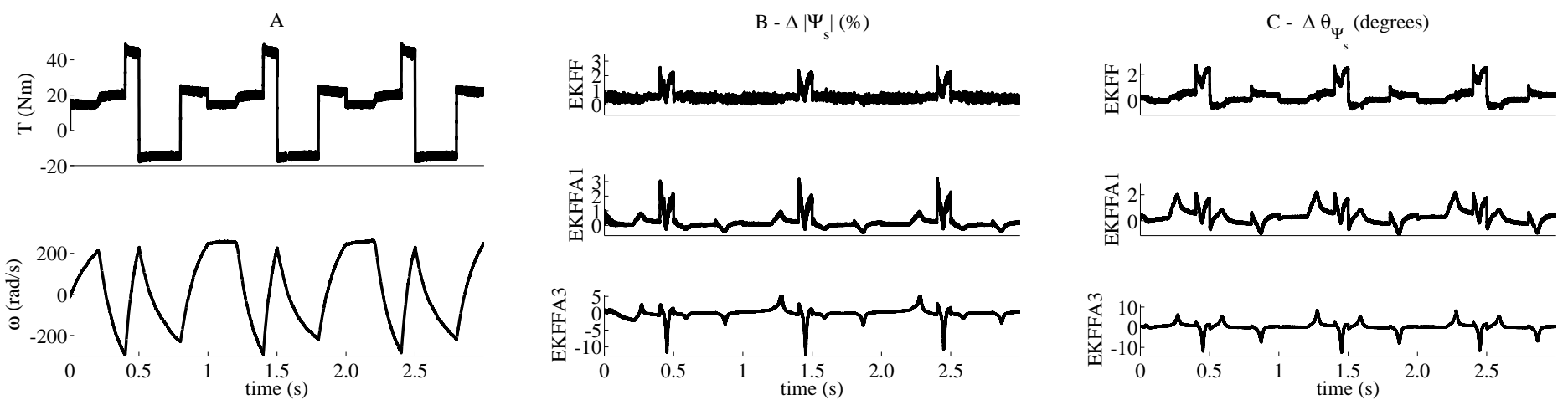

Figure 4: Dynamic operation of SPMSM with saturation. A: Torque and speed B: percentage flux magnitude error C: flux angle error

In reality however many SPMSMs do not necessarily have a symmetrical non-salient construction which can result in a different $L_{d}$ and $L_{q}$. Besides this physical saliency, saturationinduced saliency can occur as the current in $d$ and $q$ axes is different and will saturate the iron of the motor. Finally the saturation of the iron due to the permanent magnet itself can give rise to a difference between $d$ and $q$-axis inductance for a no-load situation as well.

To study the behaviour of saturated PMSMs a broadband frequency-domain identification procedure was presented in [8], [12]. This method is an extension of the Stand Still Frequency Response (SSFR) as standardized in IEEE Std.115 and uses broadband voltage signals (mainly multisines) to excite the PMSM. The machine impedance is obtained from the current response in the frequency domain and the machine inductance is extracted with a least-squares method. The approach has been tested both with a linear amplifier [12] and an inverter [8] to provide the excitation. In figure 3 the obtained inductances $L_{d}$ and $L_{q}$ for a Siemens PMSM are depicted. Clearly the motor construction and the saturation in the $d$-axis due to the permanent magnet result in $L_{d}<L_{q}$. Besides the no-load difference in inductance there is also an expected, but appreciable difference when either axis saturates. In this light three questions arise:

- How is the stator flux estimation influenced by modelling $L_{d}=L_{q}=L_{s}$ without saturation?

- How is the parameter estimation influenced by this?
- Can the stator flux and/or parameter estimation be improved by using an anisotropic model in the EKF?

To study the influence of the assumptions $\left(L_{d}=L_{q}=L_{s}\right)$ used in the EKF, simulations were performed where EKFC and EKFF estimate the stator flux vector of a motor with anisotropy and saturation. Motor parameters are given in Table V; saturation and anisotropy information is given in figure 3. For the highly dynamical operating conditions (loads up to 3 times nominal torque, load and speed reversing) of figure $4 \mathrm{~A}$, the errors on the stator flux vector magnitude (4B) and angle (4C) obtained with EKFF are shown (EKFF would be even more sensitive to variations of $L_{s}$ then EKFC, as discussed before). Clearly EKFF remains stable and the influence of assuming $L_{d}=L_{q}=L_{s}=$ $10 \mathrm{mH}$ is rather small (less then $3 \%$ in amplitude, and maximal 2 electrical degrees in angle).

When the parameter $L_{s}$ is estimated, for EKFFA1 and EKFFA3, the stator flux estimation remains stable as shown in figure 4. For EKFA1 the errors on flux magnitude and angle are again small, notice the steady-state improvement between EKFA1 and EKFF: if there is no transient the error becomes almost zero after some time for EKFFA1. In figure 5 is shown how the $L_{s}$-estimation converges after each transient to the average value of $L_{d}$ (even when, due to temporary flux weakening operation, $L_{q}<L_{d}$ ) For EKFFA3 $R_{s}$ and $\Psi_{f}$ are additionally estimated. In figure 5 it is clear that the error in $L_{s}$ influences the other parameter estimations as well, showing the difficulty to select the $\mathbf{Q}$ matrix. 


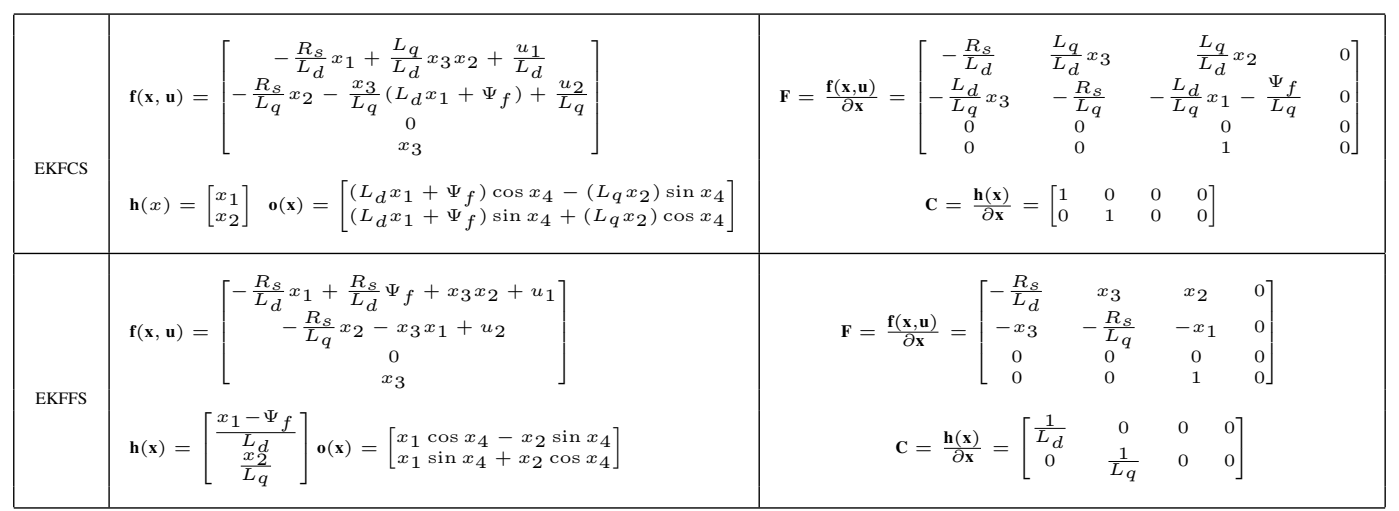

Table III: Equations for EKFCS and EKFFS with $\alpha \beta \rightarrow d q$ outside the EKF (4.2)

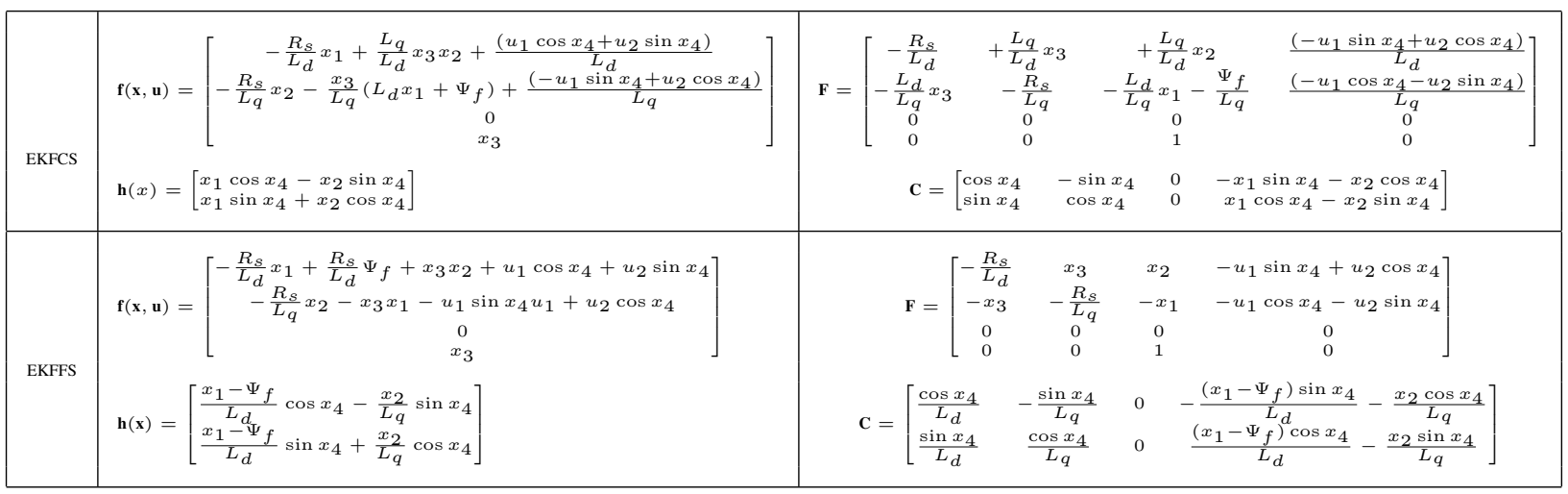

Table IV: Equations for EKFCS and EKFFS with $\alpha \beta \rightarrow d q$ in the EKF (4.3)
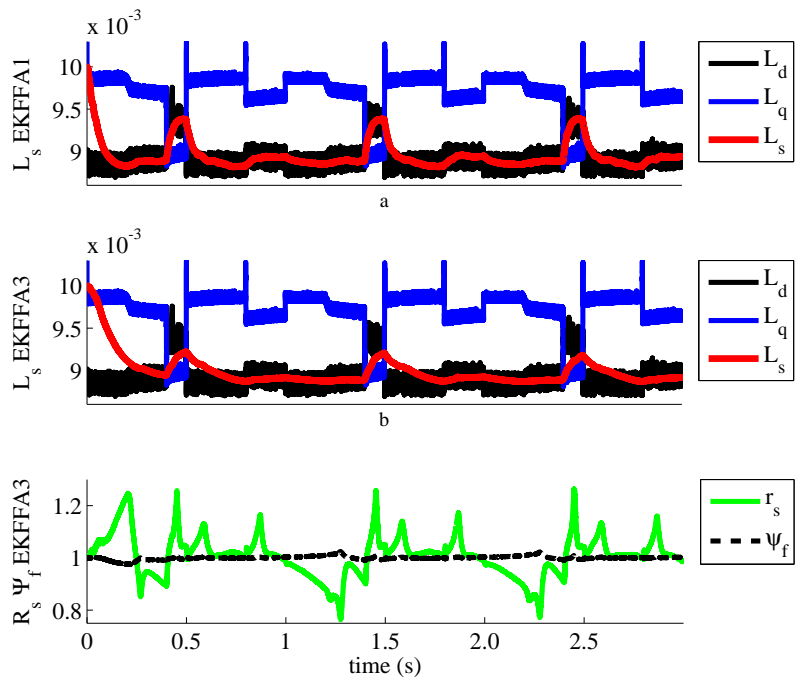

Figure 5: Evolution of the estimated parameters in the EKF

The resulting loss of estimation precision can be seen in figure 4 , with errors up till $10 \%$ and 10 electrical degrees. Overall it can be concluded that the influence of the motor anisotropy and saturation is not too dramatic for the EKF. However this depends strongly on the parameter values for particular motors, this PMSM has a small $L_{s}$ for the given $\Psi_{f}$.

\section{EKF for anisotropic motors}

Even though the influence of anisotropy can be rather small for certain motors, it can be more important for others. Furthermore an extension of these results to interior permanent magnet synchronous motors (IPMSM) is desired. The use of EKFs for IPMSMs in literature is very limited and always focused on the estimation of the rotor position, not the stator flux vector.

As discussed in [1] executing the EKF for IPMSMs in a stationary $\alpha \beta$ reference frame is very difficult. As such the EKF for IPMSMs the motor model departs from equations 6-7 in a $d q$ reference frame. This means that the state vector for the EKFCversion (EKFCS) and EKFF-version (EKFFS) are respectively:

$$
\begin{aligned}
& \mathbf{x}=\left[\begin{array}{llll}
x_{1} & x_{2} & x_{3} & x_{4}
\end{array}\right]^{T}=\left[\begin{array}{lll}
i_{d} & i_{q} & \omega \theta
\end{array}\right]^{T} \\
& \mathbf{x}=\left[\begin{array}{llll}
x_{1} & x_{2} & x_{3} & x_{4}
\end{array}\right]^{T}=\left[\begin{array}{lll}
\Psi_{d} & \Psi_{q} & \omega \theta
\end{array}\right]^{T}
\end{aligned}
$$

The measured inputs and outputs however are obtained in the stationary $\alpha \beta$ reference frame. As such there is a choice to perform the change of reference frame for $\mathbf{u}, \mathbf{y}$, and $\mathbf{z}$ either outside of the EKF or inside.

\subsection{EKF with $u, y$, and $z$ in the $d q$ reference frame}

A classical approach [1] is to leave the rotational transformation out of the EKF, the equations are given in Table III. In that case the input $\mathbf{u}$ and outputs $\mathbf{y}, \mathbf{z}$ used in the EKF are in the $d q$ reference frame:

$$
\begin{aligned}
& \mathbf{u}=\left[\begin{array}{ll}
u_{1} & u_{2}
\end{array}\right]^{T}=\left[\begin{array}{ll}
V_{d, m} & V_{q, m}
\end{array}\right]^{T} \\
& \mathbf{y}=\left[\begin{array}{ll}
y_{1} & y_{2}
\end{array}\right]^{T}=\left[\begin{array}{ll}
i_{d} & i_{q}
\end{array}\right]^{T} \\
& \mathbf{z}=\left[\begin{array}{ll}
z_{1} & z_{2}
\end{array}\right]^{T}=\left[\begin{array}{ll}
i_{d, m} & i_{q, m}
\end{array}\right]^{T}
\end{aligned}
$$

This change of reference frame is based on the estimated rotor position $\theta$ from the EKF. However, the estimated rotor position $\theta$ is not really used in the EKF and follows only from the estimated speed $\omega$. This means that a small remaining error in the speed will give rise to an increasing error in the position. As such the 

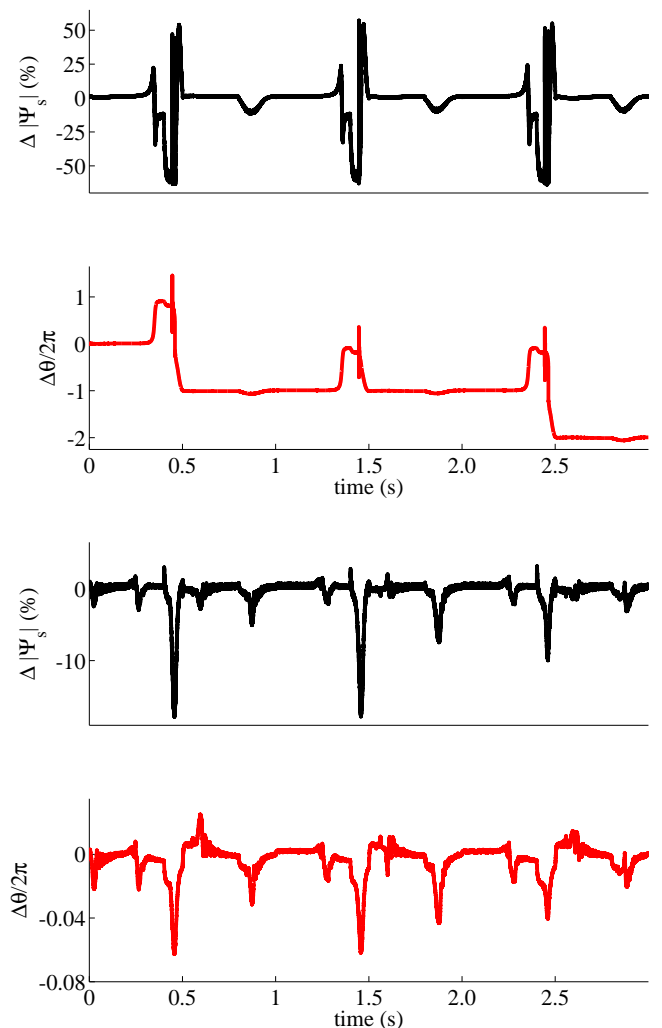

Figure 6: Top: $\alpha \beta \rightarrow d q$ outside EKF. Bottom: $\alpha \beta \rightarrow d q$ inside EKF

change of reference frame becomes more increasingly incorrect until the estimation 'slips' and reconverges on a correct solution $[\omega, \theta \pm 2 k \pi]$ instead of $[\omega, \theta]$. In figure 6 the resulting error in the stator flux magnitude (up to more then $50 \%$ ) is shown together with the evolution of $\frac{\Delta \theta}{2 \pi}$ for the load cycle of figure 4 .

\subsection{EKF with $u, y$, and $z$ in the $\alpha \beta$ reference frame}

The equations in Table III are valid when executing the rotational transformation inside the EKF (the expression for $\mathbf{o}(\mathbf{x})$ remains the same as previously). In that case the input $\mathbf{u}$ and outputs $\mathbf{y}, \mathbf{z}$ used in the EKF are in the $\alpha \beta$ reference frame:

$$
\begin{aligned}
& \mathbf{u}=\left[\begin{array}{ll}
u_{1} & u_{2}
\end{array}\right]^{T}=\left[\begin{array}{ll}
V_{\alpha, m} & V_{\beta, m}
\end{array}\right]^{T} \\
& \mathbf{y}=\left[\begin{array}{ll}
y_{1} & y_{2}
\end{array}\right]^{T}=\left[\begin{array}{ll}
i_{\alpha} & i_{\beta}
\end{array}\right]^{T} \\
& \mathbf{z}=\left[\begin{array}{ll}
z_{1} & z_{2}
\end{array}\right]^{T}=\left[\begin{array}{ll}
i_{\alpha, m} & i_{\beta, m}
\end{array}\right]^{T}
\end{aligned}
$$

When inspecting the equations in Table III and Table IV, it is clear that in the second case much more information about $\theta$ is available and a better correction on $\theta$ can be performed. The downside is of course the increased computational complexity. In figure 6 clearly the error in the stator flux magnitude is much smaller and the error $\frac{\Delta \theta}{2 \pi}$ is quickly corrected.

\subsection{Added parameters}

To obtain better estimations with varying parameters, augmenting the state vector exists as a possibility here as well. However, one should take into account that estimating the inductances means already an increase of 2 for the order in the case of EKFFS. For EKFCS there are additional problems: due to the $\frac{L_{d}}{L_{q}}$ and $\frac{L_{d}}{L_{q}}$ factors a division of two state variables is present (even when we estimate $\frac{1}{L_{q}}$ and $\frac{1}{L_{d}}$. Due to the partial differentiation this results in expressions for the $\mathbf{F}$ and $\mathbf{C}$ that are much more complex and are prohibitive for an actual FPGA implementation.

\section{Conclusions}

In this paper the use of several formulations of an Extended Kalman Filter to estimate the PMSM stator flux vector has been studied. Besides formulating different versions, the influence of incorrect motor parameters and modelling inaccuracies, such as anisotropy and saturation, have been discussed. For SPMSM it is shown that using an EKF with isotropic motor model works well, even when the motor is anisotropic and saturates. When estimating motor parameters in the EKF $\Psi_{f}$, potentially together with $L_{s}$, is the best choice. For motors with important saliency (IPMSMs) suitable versions of the EKF are presented. Executing the change of reference frame inside the EKF is preferable.

\section{Acknowledgment}

T. Vyncke wishes to thank the Research Foundation-Flanders for his $\mathrm{Ph}$. D. fellowship of the Research Foundation - Flanders (FWO). This work was supported by the GOA project BOF 07/GOA/006. The research was performed as part of the Interuniversity Attraction Poles programme IUAP P6/21 financed by the Belgian government.

\begin{tabular}{|ll|ll|ll|}
\hline$R_{s}$ & $1 \Omega$ & $L_{s}$ & $10.4 \mathrm{mH}$ & $J$ & 48 \\
$\Psi_{f}$ & $0.35 \mathrm{~Wb}$ & $N_{p}$ & 4 & $T_{n}^{-4} \mathrm{kgm}^{2}$ & $16.9 \mathrm{Nm}$ \\
\hline
\end{tabular}

\section{References}

[1] S. Bolognani, L. Tubiana, and M. Zigliotto. EKF -based sensorless IPM synchronous motor drive for flux-weakening applications. IEEE Trans. Ind. Applicat., 39(3):768-775, May/June 2003.

[2] S. Bolognani, L. Tubiana, and M. Zigliotto. Extended Kalman filter tuning in sensorless PMSM drives. IEEE Trans. Ind. Applicat., 39(6):1741-1747, Nov./Dec. 2003.

[3] V. Comnac, M. Cernat, F. Moldoveanu, and I. Draghici. Sensorless speed and direct torque control of surface permanent magnet synchronous machines using an extended kalman filter. In Conf. Proc. 9th Mediterranean Conference on Control and Automation (MED'01), page 6, Dubrovnik, Croatia, June 27-29, 2001.

[4] A. Llor, J. Rétif, X. Lin-Shi, and S. Arnalte. Direct stator flux linkage control technique for a permanent magnet synchronous machine. In Conf. Rec. IEEE Annual Power Electronics Specialists Conference, volume 1, pages 246-250, June 15-19, 2003.

[5] J. Luukko, M. Niemelä, and J. Pyrhönen. Estimation of the flux linkage in a direct-torque-controlled drive. IEEE Trans. Ind. Electron., 50(2):283-287, Apr. 2003.

[6] M. F. Rahman, L. Zhong, M. E. Haque, and M. Rahman. A direct torque-controlled interior permanent-magnet synchronous motor drive without a speed sensor. IEEE Trans. Energy Conversion, 18(1):17-22, Mar. 2003.

[7] I. Takahashi and T. Noguchi. A new quick-response and highefficiency control strategy of an induction motor. IEEE Trans. Ind. Applicat., 22(5):820-827, Sept./Oct. 1986.

[8] T. Vandoorn, F. M. De Belie, T. J. Vyncke, J. A. Melkebeek, and P. Lataire. Generation of multisinusoidal test signals for the identification of synchronous-machine parameters by using a voltage-source inverter. IEEE Trans. Ind. Electron., 57, Issue 1:430-439, Jan. 2010.

[9] P. Vas. Sensorless Vector and Direct Torque Control, pages 122178. Oxford University Press, New York, 1998.

[10] T. J. Vyncke, R. K. Boel, and J. A. Melkebeek. A comparison of stator flux linkage estimators for a direct torque controlled PMSM drive. In Conf. Proc. Industrial Electronics Conference (IECON 2009), page 6, Porto, Portugal, Nov.3-5, 2009.

[11] T. J. Vyncke, R. K. Boel, and J. A. Melkebeek. On extended kalman filters with augmented state vectors for the stator flux estimation in SPMSMs. In Conf. Proc. Applied Power Electronics Conference, page 8, Palm Springs, CA., USA, Feb. 21-25, 2010.

[12] T. J. Vyncke, F. M. De Belie, R. K. Boel, J. A. Melkebeek, Y. Cheng, and P. Lataire. Identification of PM synchronous machines in the frequency domain by broadband excitation. In Conf. Proc. Power Electronics, Electrical Drives, Automation and Motion, pages 1253 - 1258, Ischia, Italy, June 11-13, 2008. 